\title{
COMPREENSÃO DAS MANIFESTAÇÕES NEUROLÓGICAS INDUZIDAS POR INFECÇÕES PELO NOVO CORONÁVÍRUS: UMA REVISÃO INTEGRATIVA
}

\section{UNDERSTANDING NEUROLOGICAL MANIFESTATIONS INDUCED BY INFECTIONS BY THE NEW CORONAVIRUS: AN INTEGRATIVE REVIEW}

\author{
Letícia Pereira Felipe ${ }^{1} *$ Arthur Castro de Lima $^{2} *$ Karina Gonzaga da Costa ${ }^{3}$ N'ghalna da Silva ${ }^{4}$ ' \\ Davide Carlos Joaquim ${ }^{5} *$ Daniel Freire de Sousa ${ }^{6} *$ Ana Caroline Rocha de Melo Leite $^{7}$
}

\begin{abstract}
RESUMO
Objetivo: Compreender as manifestações clínicas neurológicas apresentadas por pacientes com coronavírus da Síndrome Respiratória Aguda Grave 2 (SARS-CoV-2)/Doença Coronavírus 19 (COVID-19). Métodos: Trata-se de revisão integrativa, conduzida em junho de 2020, com base na pergunta norteadora "Quais as manifestações neurológicas apresentadas por pacientes com SARS-CoV-2/COVID-19?, elaborada a partir da estratégia PICO. A combinação de palavras "SARS-CoV-2" AND "COVID-19" AND "neurologic manifestations" foi utilizada na busca em bases de dados Cumulative Index to Nursing and Allied Health Literature (CINAHL), National Library of Medicine (PubMed), Scopus e Web of Science. Resultados: Dos 6 artigos incluídos, 4 constavam na PubMed e todos tinham sido publicados em periódicos internacionais. Dentre as manifestações observadas, as disfunções olfativas e gustativas foram as que se destacaram. Outras manifestações observadas envolveram desde a mialgia, dor de cabeça e tontura à encefalite e Síndrome de Guillain-Barré. Conclusão: Manifestações neurológicas estão presentes em pacientes com SARS-CoV2/COVID-19, incluindo aqueles não hospitalizados, destacando as disfunções olfativas e gustativas. Estas podem estar associadas a um quadro de COVID-19 precoce e severo, sendo, portanto, importante o seu reconhecimento na infecção. Apesar da ocorrência dessas manifestações, ainda se faz necessária a investigação dos mecanismos patogênicos e progressão da doença no contexto do Sistema Nervoso Central (SNC) e Periférico (SNP).
\end{abstract}

Palavras-chave: Manifestações Neurológicas; Infecções por Coronavírus; Enfermagem; Pandemia; Revisão.

\section{ABSTRACT}

Objective: To understand the clinical neurological manifestations of patients with Coronavirus of Severe Acute Respiratory Syndrome 2 (SARS-CoV-2)/Coronavirus 19 (COVID-19). Methods: This integrative review was conducted in June 2020, based on the guiding question "What are the neurological manifestations presented by patients with SARS-CoV-2/COVID-19?, based on the PICO strategy. The combination of words "SARS-CoV-2" AND "COVID-19" AND "neurologic manifestations" was used in the search in databases Cumulative Index to Nursing and Allied Health Literature (CINAHL), National Library of Medicine (PubMed), Scopus, and Web of Science. Results: Of the six articles included, four were in PubMed, and all had been published in international journals. Among the manifestations observed, olfactory and gustatory disorders stood out. Other manifestations observed ranged from myalgia, headache, and dizziness to encephalitis and Guillain-Barré syndrome. Conclusion: Neurological manifestations are present in patients with SARS-CoV-2/COVID-19, including those not hospitalized, highlighting olfactory and taste disorders. These may be associated with early and severe COVID-19, and its recognition in infection is therefore essential. Despite the occurrence of these manifestations, it is still necessary to investigate the pathogenic mechanisms and progression of the disease in the context of the Central Nervous System (CNS) and Peripheral (PNS). Keywords: Neurological Manifestations; Coronavirus Infections; Nursing; Pandemics; Review.

\footnotetext{
1 Universidade da Integração Internacional da Lusofonia Afro-Brasileira (UNILAB), Redenção, Brasil. ORCID https://orcid.org/0000-0003-2551-9143

2 Universidade da Integração Internacional da Lusofonia Afro-Brasileira (UNILAB), Redenção, Brasil. ORCID https://orcid.org/0000-0003-1826-2247

3 Universidade da Integração Internacional da Lusofonia Afro-Brasileira (UNILAB), Redenção, Brasil. ORCID https://orcid.org/0000-0002-4127-0424

4 Universidade da Integração Internacional da Lusofonia Afro-Brasileira (UNILAB), Redenção, Brasil. ORCID https://orcid.org/0000-0003-2937-6960

${ }^{5}$ Universidade Federal do Ceará (UFC), Fortaleza, Brasil. ORCID https://orcid.org/0000-0003-0245-3110

6 Universidade da Integração Internacional da Lusofonia Afro-Brasileira (UNILAB), Redenção, Brasil. ORCID https://orcid.org/0000-0002-2333-5343

7 Universidade da Integração Internacional da Lusofonia Afro-Brasileira (UNILAB), Redenção, Brasil ORCID https://orcid.org/0000-0002-9007-7970
} 


\section{INTRODUÇÃO}

Identificados desde a década de 1960, os coronavírus são RNA vírus envelopados, pertencentes à ordem Nidovirales, família Coronaviridae e subfamília Coronavirinae, cujo aspecto na microscopia eletrônica se assemelha a uma coroa (latim - corona) ${ }^{(1)}$. Capazes de infectar animais, aves e seres humanos, eles promovem distúrbios respiratórios, gastroentéricos, hepáticos e neurológicos ${ }^{(2,3)}$. Atualmente, sete cepas de coronavírus já foram reconhecidas como patógenos em humanos $^{(4)}$.

Em 2002, a humanidade vivenciou um surto provocado pelo coronavírus da Síndrome Respiratória Aguda Grave (SARS$\mathrm{CoV})$, o qual tem, como hospedeiro primário/definitivo, o morcego ${ }^{(5)}$. Iniciada em Guandong, no sul da China, a infecção se propagou para o Sudeste Asiático, Europa, África do Sul e América do Norte ${ }^{(6,7)}$.

Em termos epidemiológicos, em julho de 2003, a Organização Mundial da Saúde (OMS) registrou 8.437 casos de SARS e 813 óbitos $^{(6)}$. Embora desde aquele ano não tenha sido relatado qualquer outro surto da doença $^{(8)}$, inexiste um conhecimento adequado sobre a patologia do SARS-CoV, prejudicando a escolha do tratamento e desenvolvimento de vacinas ${ }^{(5)}$.

Após dez anos do SARS-CoV, um novo coronavírus foi identificado, tendo o dromedário como hospedeiro definitivo e, como primeiro local de registro da doença, Jeddah, na Arábia Saudita $^{(9)}$. Desde seu primeiro relato, casos da doença, denominada Síndrome Respiratória do Oriente Médio (MERS), foram notificados em toda a Península Arábica, Ásia, Europa, África e Estados Unidos ${ }^{(9)}$.

Especificamente, a MERS é uma condição desencadeada pelo MERS-CoV, cuja manifestação varia da forma assintomática à pneumonia grave, SARS, falência de múltiplos órgãos e morte ${ }^{(10)}$. Segundo a literatura, MERS-CoV foi considerado uma ameaça epidêmica à saúde pública em decorrência dos milhões de peregrinos dos 184 países que se deslocam para a Arábia Saudita ${ }^{(9)}$. O MERS-CoV ainda está circulando ${ }^{(7)}$, tendo sido notificado, em janeiro de 2020, um total de 2.519 ocorrências da infecção e 866 óbitos, especialmente na Península Arábica ${ }^{(4)}$.

À semelhança do SARS-CoV, o MERS-CoV infecta inicialmente o trato respiratório inferior ${ }^{(11)}$, podendo ser transmitido via contato com uma pessoa infectada ou superfície e objeto contaminados. Sua patologia não é clara, dificultando a terapia e desenvolvimento de vacinas ${ }^{(5)}$.

Em dezembro de 2019, surgiu em Wuhan, na China, um surto provocado por um novo tipo de coronavírus, denominado, pelo Comitê Internacional de Taxonomia de Vírus 
(ICTV), de SARS-CoV-2. Poucos meses após, o SARS-CoV-2 se alastrou pelos cinco continentes, induzindo a OMS, em março de 2020, a declará-lo como emergência de saúde pública internacional devido a sua alta capacidade de disseminação e transmissão assintomática $^{(12)}$. Atualmente, há forte evidência de que o vírus foi oriundo de $\operatorname{morcegos}^{(13)}$.

De acordo com os dados da $\mathrm{OMS}^{(14)}$, até 25 de abril de 2021, foram notificados 146.054.107 casos da Doença Coronavírus 19 (COVID-19), infecção causada pelo SARSCoV-2, 3.092.410 óbitos e um maior acometimento na América. Conforme a OMS, Estados Unidos, Índia e Brasil figuram entre os países mais afetados, com 31.656.636, 16.960.172 e 14237.078 casos confirmados da doença, respectivamente.

No tocante ao SARS-CoV-2, esse, embora apresente muitas características genéticas semelhantes ao MERS-CoV, expressa elevada homologia com o SARS do morcego e SARS-CoV ${ }^{(15-16)}$. À semelhança do SARS-CoV e MERS, ele pode ser altamente fatal, acometendo inicialmente $\mathrm{o}$ trato respiratório superior e inferior ${ }^{(11)}$. Sua transmissão ocorre via gotículas provenientes das narinas ou cavidade oral de pessoas infectadas, exteriorizadas no ato de falar, expirar, tossir ou espirrar. O vírus pode ser transmitido indiretamente, por meio do contato com objetos contaminados ${ }^{(17)}$ e via transplacentária ${ }^{(18)}$.

Quanto ao seu mecanismo de ação, o SARS-CoV-2 liga-se à Enzima Conversora de Angiotensina 2 (ECA-2) (expressa no tecido epitelial do trato respiratório, endotélio, rim, parênquima pulmonar e células do intestino delgado $)^{(18-20)}$, por meio da sua proteína Spike (S), fenômeno seguido pela endocitose e ativação dessa proteína pela serina protease transmembrana 2 (TMPRSS2) (enzima ligada à membrana celular localizada nas proximidades da ECA-2) ${ }^{(21)}$.

A clivagem proteolítica da proteína $S$ pela TMPRSS2 pode também ocorrer diretamente, promovendo a fusão entre a membrana viral e a plasmática, liberando o RNA do vírus no citoplasma. O RNA presente no citoplasma ou endossomo ativará sistemas de detecção de ácido nucleico, como receptores Toll-like, desencadeando a ativação de fator nuclear $-\kappa \beta(\mathrm{NF}-\kappa \beta)$ e produção de mediadores inflamatórios ${ }^{(21)}$.

Nos casos de infecção pelo SARSCoV-2, seus sintomas têm variado desde manifestações leves a severas ${ }^{(11)}$, tendo, como apresentação típica, febre, tosse, mialgia, fadiga e pneumonia ${ }^{(22)}$. Os sintomas leves compreendem desde febre, tosse e falta de ar à cefaleia, perda do olfato e paladar e dor de garganta. Os sintomas graves incluem pneumonia, SARS e sepse ${ }^{(11)}$. Dados epidemiológicos apontam que $80 \%$ da 
população contaminada apresenta quadros de pneumonia atípica de leve à moderada, $15 \%$ evolui para pneumonia grave e 5\% pode desenvolver SARS ${ }^{(23)}$.

Com relação aos sintomas e sinais neurológicos, pela similaridade entre SARSCoV-2 e outros beta coronavírus, não se espera esse tipo de manifestação e possíveis complicações $^{(24)}$. Entretanto, têm sido cada vez mais frequentes os relatos dessas manifestações $^{(25)}$, as quais parecem ocorrer em aproximadamente $36 \%$ dos pacientes $^{(26)}$. Elas incluem cefaleia, tontura, eventos cerebrovasculares, anosmia, ageusia e encefalopatia $^{(25,27)}$. Apesar dessas ocorrências, os estudos relacionados à infecção pelo SARS-CoV-2 do Sistema Nervoso Central (SNC) e Periférico (SNP) são limitados ${ }^{(26)}$.

Nesse sentido, a literatura tem sugerido a atuação da desregulação imune induzida pelo SARS-CoV-2, marcada pela depleção de células $\mathrm{T}$ e excesso de produção de citocinas, na promoção de sintomas neurológicos ${ }^{(25,28)}$. Embora ainda não confirmada a neuroinvasividade, sugere-se a disseminação hematogênica e retrógada neuronal como vias de acesso do vírus ao $\mathrm{SNC}^{(28)}$.

Dessa forma, independentemente dos meios utilizados pelo SARS-CoV-2 na indução de possíveis sinais e sintomas neurológicos, faz-se necessária a conscientização dessa realidade como tentativa de reconhecer, tratar e gerir complicações neurológicas potencialmente fatais $^{(27)}$.

Nessa perspectiva, a Enfermagem se destaca pelo papel relevante que desempenha no enfrentamento da COVID-19, prestando assistência nos diferentes níveis de atenção à saúde ao indivíduo, família e comunidade ${ }^{(27)}$. Além do que, essa categoria profissional estabelece mais facilmente vínculo com o paciente e oferece auxílio a ele durante 24 horas $^{(27)}$, possibilitando a detecção das mais diversas apresentações da doença, inclusive no âmbito do SNC e SNP.

Diante do exposto, o estudo teve como objetivo compreender as manifestações neurológicas apresentadas por pacientes com SARS-CoV-2/COVID-19.

\section{MÉTODOS}

Trata-se de uma revisão integrativa, método da Prática Baseada em Evidências (PBE) que contribui para o aprofundamento do conhecimento sobre um determinado assunto. Esse método permite reunir e sintetizar resultados de estudos primários, de forma sistemática e ordenada, facilitando a incorporação de evidências para tomada de decisão e indicando possíveis lacunas a serem esclarecidas em novas pesquisas na área ${ }^{(29)}$.

A condução dessa revisão seguiu seis etapas, a saber: - identificação do tema e elaboração da questão de pesquisa; - 
estabelecimento de critérios para inclusão e exclusão de estudos; - categorização dos estudos; - avaliação dos estudos incluídos na revisão integrativa; - interpretação dos resultados; - síntese do conhecimento dos principais resultados evidenciados na análise dos artigos incluídos ${ }^{(29,30)}$.

A questão norteadora foi elaborada com base na estratégia PICO, acrônimo no idioma inglês, cujo significado corresponde a "paciente, intervenção, comparação e resultados". Com base nessa estratégia, tida como um recurso relevante para a formulação de questões de pesquisa e busca de evidências na literatura ${ }^{(30,31)}$, a revisão atribuiu, para a letra "P", paciente com SARS-CoV2/COVID-19, "I" e "C" não foram aplicados e "O" foi associado a manifestações neurológicas. Assim, obteve-se a seguinte pergunta norteadora: Quais as manifestações neurológicas apresentadas por pacientes com SARS-CoV-2/COVID-19?

A busca e análise dos artigos ocorreram em junho de 2020, via consulta ao Portal de Periódico da Coordenação de Aperfeiçoamento de Pessoal de Nível Superior (CAPES)/Ministério da Educação (MEC), nas seguintes bases de dados: Cumulative Index to Nursing and Allied Health Literature (CINAHL), National Library of Medicine (PubMed), Scopus e Web of Science.
Para as buscas dos artigos, foi utilizado o descritor controlado em inglês "Neurologic manifestations", indexado nos descritores em Ciências da Saúde (DeCS), e as palavras-chave "SARS-CoV-2" e "COVID-19". Esses termos foram combinados da seguinte forma: "SARS-CoV2" AND “COVID-19” AND "neurologic manifestations".

Foram considerados, como critérios de inclusão, artigos disponíveis na íntegra, nos idiomas português, inglês e espanhol e que respondessem à questão norteadora. Os critérios de exclusão adotados compreenderam: publicações repetidas, dissertações, estudos de casos e artigos que não se relacionavam com o objetivo da revisão. A seleção dos estudos seguiu as recomendações do método Preferred Reporting Items for Systematic Reviews and Meta-Analyses - PRISMA ${ }^{(32)}$.

Para a coleta e análise de dados dos artigos selecionados, foi elaborado um instrumento baseado no estudo de Ursi ${ }^{(33)}$, com os seguintes itens: codificação dos artigos, autores, título da publicação, país da publicação, periódico, base de dados, objetivo, tipo de estudo, nível de evidência e resultados. Essas etapas foram realizadas por quatro acadêmicos do Curso de Enfermagem de uma universidade brasileira de cunho internacional, de forma independente e de 
modo a permitir uma maior confiabilidade na avaliação dos estudos selecionados.

Foi realizada a classificação das evidências dos artigos, fundamentada na qualidade ou força da evidência, que pode ser caracterizada nos seguintes níveis: nível I revisão sistemática ou metanálise; nível II estudo randomizado controlado; nível III estudo controlado sem randomização; nível IV - estudo caso-controle ou estudo de coorte; nível V - revisão sistemática de estudos qualitativos ou descritivos; nível VI estudo qualitativo ou descritivo; nível VII opinião ou consenso ${ }^{(34)}$.
Mediante aplicação da estratégia de busca, foram encontrados 1.679 trabalhos, dos quais 986 haviam sido publicados em 2020. Desses, 481 estavam no idioma inglês, espanhol ou português. Após a leitura do título e resumo, foram excluídas 460 publicações por não se relacionarem com o objetivo da revisão e 7 por duplicidade. Dos 14 artigos lidos na íntegra, 5 foram excluídos por serem estudo de caso e dissertação e 3 por não responderem à pergunta norteadora. Assim, a revisão foi composta por 6 artigos (Figura 1).

\section{RESULTADOS}

Figura 1 - Fluxograma de identificação do processo de seleção dos estudos para compor a revisão integrativa. Redenção - CE, Brasil, 2020

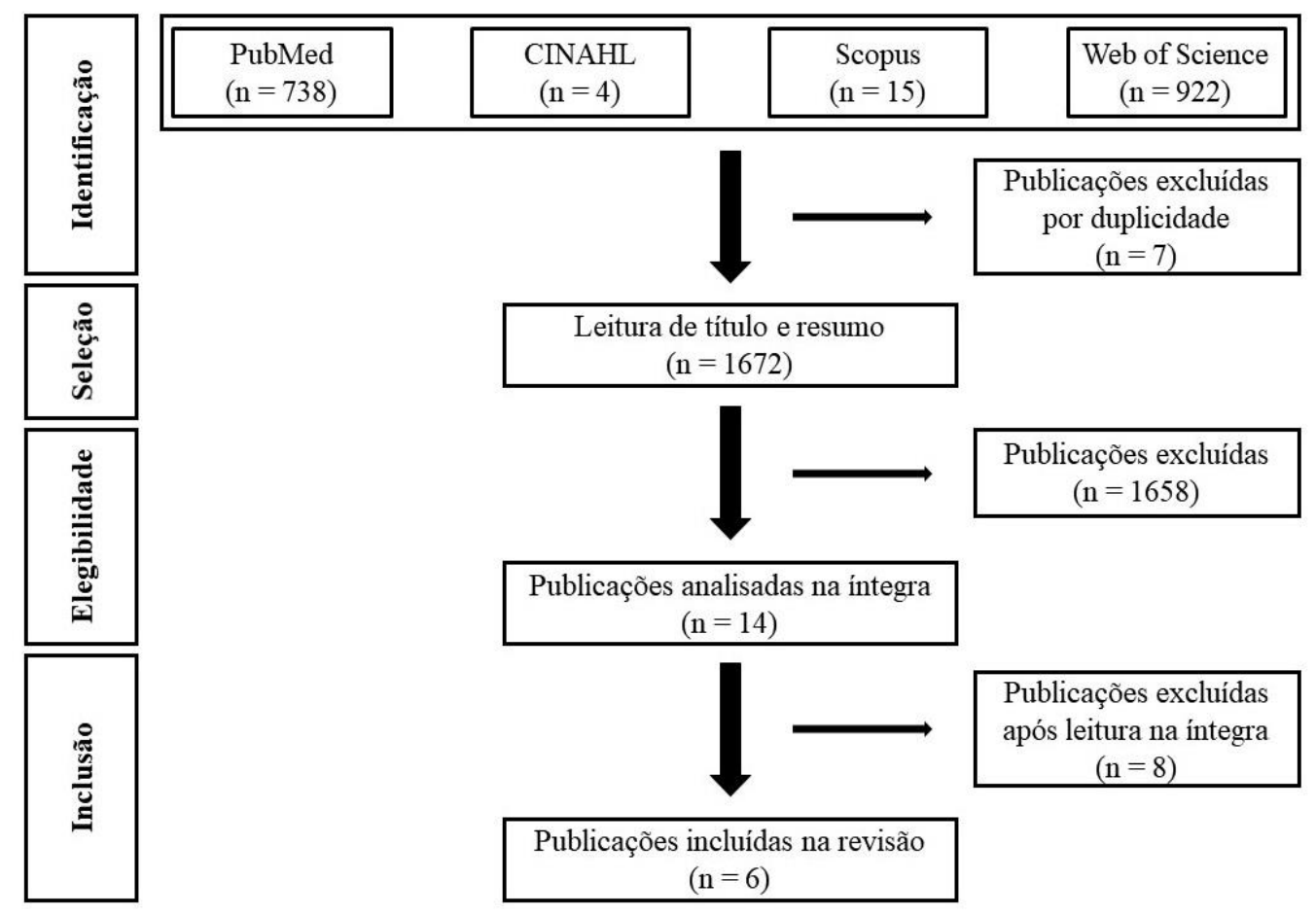

Fonte; Os autores 
Das publicações incluídas, a maioria constava na base de dados PubMed $(n=4)$ e tinha sido desenvolvida na Itália $(\mathrm{n}=4)$. Todos os artigos estavam no idioma inglês e tinham sido publicados em periódicos internacionais, especialmente na revista European Archives of Oto-RhinoLaryngology $(\mathrm{n}=3)$ (Tabela 1).

Tabela 1 - Caracterização das publicações incluídas na revisão, de acordo com o número, base de dados, título, país e periódico. Redenção - CE, Brasil, 2020.

\begin{tabular}{|c|c|c|c|c|}
\hline $\mathrm{N}^{\mathrm{o}}$ & $\begin{array}{l}\text { Base de } \\
\text { Dados }\end{array}$ & Título da Publicação & $\begin{array}{c}\text { País da } \\
\text { Publicação }\end{array}$ & Periódico \\
\hline 1 & PubMed & $\begin{array}{l}\text { Neurologic manifestations in } \\
\text { hospitalized patients with } \\
\text { COVID-19: the ALBACOVID } \\
\text { registry }\end{array}$ & Espanha & Neurology \\
\hline 2 & PubMed & $\begin{array}{l}\text { New onset of loss of smell or } \\
\text { taste in household contacts of } \\
\text { home-isolated SARS-CoV-2- } \\
\text { positive subjects }\end{array}$ & Itália & $\begin{array}{c}\text { European } \\
\text { Archives of Oto- } \\
\text { Rhino- } \\
\text { Laryngology }\end{array}$ \\
\hline 3 & PubMed & $\begin{array}{l}\text { Olfactory and } \text { gustatory } \\
\text { dysfunctions in } 100 \text { patients } \\
\text { hospitalized for COVID-19: sex } \\
\text { differences and recovery time in } \\
\text { real-life }\end{array}$ & Itália & $\begin{array}{c}\text { European } \\
\text { Archives of Oto- } \\
\text { Rhino- } \\
\text { Laryngology }\end{array}$ \\
\hline 4 & PubMed & $\begin{array}{l}\text { Subjective neurological symptoms } \\
\text { frequently occur in patients whith } \\
\text { SARS-CoV-2 infection }\end{array}$ & Itália & $\begin{array}{l}\text { Brain,Behavior, } \\
\text { and Immunity }\end{array}$ \\
\hline 5 & $\begin{array}{l}\text { Web of } \\
\text { Science }\end{array}$ & 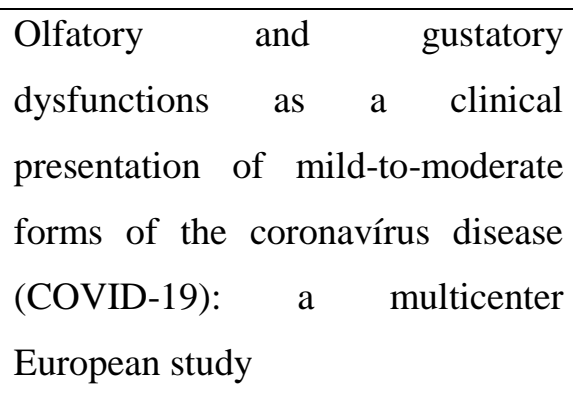 & $\begin{array}{l}\text { Itália, } \\
\text { Bélgica e } \\
\text { Espanha }\end{array}$ & $\begin{array}{c}\text { European } \\
\text { Archives of Oto- } \\
\text { Rhino- } \\
\text { Laryngology }\end{array}$ \\
\hline 6 & Web of & Olfatory dysfunction and sinonasal & Suíça & Otolaryngology- \\
\hline
\end{tabular}




\begin{tabular}{|l|l|l|l|c|}
\hline Science & $\begin{array}{l}\text { symptomatology in COVID-19: } \\
\text { prevalence, severity, timing, and } \\
\text { associated characteristics }\end{array}$ & Surgery \\
\hline
\end{tabular}

Fonte: Os autores

Quanto aos objetivos dos estudos, esses visaram identificar, quantificar e caracterizar as manifestações neurológicas relacionadas à infecção por SARS-CoV-2, bem como estabelecer o tempo de recuperação e gravidade dessas manifestações e associá-las a sintomas clássicos da COVID19. Em relação ao tipo de estudo e nível de evidência, a maioria das publicações eram pesquisas descritivas $(n=4)$ e todas tinham nível VI de evidência (Tabela 2).

Tabela 2 - Caracterização das publicações incluídas na revisão, de acordo com o número, objetivo, tipo de estudo e nível de evidência. Redenção - CE, Brasil, 2020.

\begin{tabular}{|l|l|c|c|}
\hline$N^{\circ}$ & \multicolumn{1}{|c|}{ Objetivo } & Tipo de Estudo & Nível de \\
Evidência & \\
\hline 1 & $\begin{array}{l}\text { Determinar se as manifestações neurológicas } \\
\text { comuns em pacientes com SARS-CoV-2 } \\
\text { hospitalizados e descrever suas principais } \\
\text { características. }\end{array}$ & Observacional e \\
retrospectivo & VI \\
\hline 2 & $\begin{array}{l}\text { Estimar a prevalência do comprometimento do } \\
\text { olfato ou paladar de pacientes com SARS-CoV- } \\
2 \text { isolados em casa levemente sintomáticos. }\end{array}$ & Transversal & VI \\
\hline 3 & $\begin{array}{l}\text { Investigar o momento da recuperação de } \\
\text { disfunções olfativas e gustativas em uma coorte } \\
\text { de pacientes com SARS-CoV-2 hospitalizados } \\
\text { em enfermarias de terapia não intensiva há um } \\
\text { mês antes da condução da pesquisa. }\end{array}$ & Descritivo & VI \\
\hline 4 & $\begin{array}{l}\text { Identificar e quantificar a ocorrência de } \\
\text { sintomas neurológicos subjetivos em pacientes } \\
\text { hospitalizado com infecção por SARS-CoV-2. }\end{array}$ & Observacional e \\
descritivo & VI \\
& & \\
& & \\
& & \\
\end{tabular}




\begin{tabular}{|l|l|l|l|}
\hline 5 & $\begin{array}{l}\text { Investigar e caracterizar a ocorrência de } \\
\text { disfunções olfativas e gustativas em pacientes } \\
\text { com infecção por SARS-CoV-2 confirmado em } \\
\text { laboratório. }\end{array}$ & Descritivo \\
\hline 6 & $\begin{array}{l}\text { Caracterizar a prevalência, o momento e a } \\
\text { gravidade da disfunção olfativa relatada pelo } \\
\text { paciente, bem como outros sintomas sinonasais } \\
\text { e sua associação com os sintomas clássicos do } \\
\text { SARS-CoV-2, como febre, tosse e falta de ar. }\end{array}$ & Descritivo \\
\hline
\end{tabular}

Fonte: Os autores

A partir da leitura minuciosa dos resultados apresentados pelos artigos, foi possível identificar as manifestações neurológicas em pacientes com SARS-CoV-2, sendo as mais relatadas as disfunções olfativas e gustativas. Outras manifestações observadas envolveram desde a mialgia, dor de cabeça e tontura à encefalite e Síndrome de Guillain-Barré (Tabela 3).

Tabela 3 - Caracterização das publicações incluídas na revisão, de acordo com o número e principais resultados. Redenção - CE, Brasil, 2020.

\begin{tabular}{|c|c|}
\hline $\mathrm{N}^{\mathrm{o}}$ & Principais Resultados \\
\hline 1 & $\begin{array}{l}\text { Dos } 841 \text { pacientes, } 57,4 \% \text { desenvolveram pelo menos um sintoma neurológico. } \\
\text { Os sintomas mais frequentes foram mialgias }(17,2 \%) \text {, dor de cabeça }(14,1 \%) \text { e } \\
\text { tontura }(6,1 \%) \text {. Os sintomas associados aos nervos cranianos foram anosmia } \\
(4,9 \%) \text { e disgeusia }(6,2 \%) \text {. Os distúrbios de consciência foram os sintomas } \\
\text { neurológicos mais repetidamente observados }(19,6 \%) \text {. Outros sintomas } \\
\text { descritos foram miopatia }(1,3 \%) \text {, disautonomia }(2,5 \%) \text {, doenças } \\
\text { cerebrovasculares }(1,7 \%) \text {, convulsões }(0,7 \%) \text {, distúrbios do movimento }(0,7 \%) \text {, } \\
\text { encefalite }(0,1 \%) \text {, Síndrome de Guillain-Barré }(0,1 \%) \text { e neurite óptica }(0,1 \%) \text {. }\end{array}$ \\
\hline 2 & $\begin{array}{l}\text { Dos } 296 \text { entrevistados que tiveram contato domiciliar com pacientes infectados } \\
\text { por SARS-CoV-2, } 74 \text { relataram perda de olfato ou paladar. Entre } 121 \\
\text { submetidos a teste sorológico, } 54 \text { tiveram o resultado positivo para COVID-19 e } \\
34 \text { apresentaram perda de olfato e paladar. Dos } 175 \text { que não se submeteram ao }\end{array}$ \\
\hline
\end{tabular}




\begin{tabular}{|c|c|}
\hline & $\begin{array}{l}\text { teste, } 67 \text { tinham sintomas comuns de infecção por SARS-CoV-2 e } 39 \\
\text { apresentaram perda de olfato e paladar. }\end{array}$ \\
\hline 3 & $\begin{array}{l}\text { Em } 100 \text { pacientes incluídos na pesquisa, } 42 \text { relataram disfunções } \\
\text { quimiossensoriais subjetivas. Destes, } 41 \text { citaram disfunção gustativa, } 29 \text { olfativa } \\
\text { e } 28 \text { ambas. Todos referiram a ocorrência desses sintomas na primeira semana } \\
\text { ou os consideraram como os primeiros sintomas observados. A duração média } \\
\text { da disfunção olfativa e gustativa foi de } 18 \text { e } 16 \text { dias, respectivamente. }\end{array}$ \\
\hline 4 & $\begin{array}{l}\text { Dos } 103 \text { pacientes com infecção confirmada por SARS-CoV-2 incluídos na } \\
\text { análise, } 94 \text { relataram pelo menos um sintoma neurológico. O comprometimento } \\
\text { do sono foi o mais frequente }(49,51 \%) \text {, seguido por disgeusia }(46,60 \%) \text {, dor de } \\
\text { cabeça }(38,83 \%) \text { e hiposmia }(37,86 \%) \text {. }\end{array}$ \\
\hline 5 & $\begin{array}{l}\text { Dos } 417 \text { pacientes, } 85,6 \% \text { e } 88,0 \% \text { referiram a presença de disfunções olfativas } \\
\text { e gustativas, respectivamente. Houve associação positiva significativa entre as } \\
\text { disfunções olfativas e gustativas. Em } 11,8 \% \text { dos casos, a disfunção olfativa } \\
\text { apareceu antes dos outros sintomas. Os escores do Questionário de Declarações } \\
\text { Negativas de Distúrbios Olfativos foram significativamente menores em } \\
\text { pacientes com anosmia em comparação com indivíduos com normosmia ou } \\
\text { hiposmia. }\end{array}$ \\
\hline 6 & $\begin{array}{l}\text { A prevalência de disfunção olfativa foi de } 61,2 \% \text { e sua gravidade se } \\
\text { correlacionou significativamente com a severidade da perda de paladar } \\
\text { vivenciada pelo paciente. } \\
\text { Em } 8,7 \% \text { dos participantes, a disfunção olfativa foi experienciada no primeiro } \\
\text { dia de SARS-CoV-2, sendo geral no terceiro dia de infecção. Dos pacientes com } \\
\text { disfunção olfativa, } 95,2 \% \text { apresentaram pelo menos um dos seguintes sintomas: } \\
\text { febre, tosse ou falta de ar. }\end{array}$ \\
\hline
\end{tabular}

Fonte: Os autores

\section{DISCUSSÃO}

Esta revisão integrativa evidenciou as manifestações neurológicas relacionadas à COVID-19, cuja importância se fundamenta, além das consequências a elas associadas, a possibilidade de corresponderem aos primeiros sinais de infecção em pacientes ditos assintomáticos ou com sintomas leves e 
de ocorrerem em indivíduos em processo de recuperação de manifestações não neurológicas há algumas semanas ${ }^{(35-38)}$. O conhecimento dessas manifestações pelos profissionais de saúde, especialmente o enfermeiro, poderá contribuir para projetar as estratégias de diagnóstico e intervenção, nos diferentes níveis de gravidade da doença, assim como avaliar as consequências clínicas da infecção ${ }^{(35)}$.

Quando avaliado o quantitativo de estudos incluídos nessa pesquisa, de acordo com a base de dados, o destaque para a PubMed ressalta o fato de ser ela a maior base de dados disponível desde 1996, sendo constituída por mais de 26 milhões de citações do Medline, periódicos de ciências da vida e livros online. Além do que, ela exibe links de acesso a textos completos nas citações da PubMed Central $^{(39)}$.

Quanto ao periódico das publicações, o fato de todos os artigos incluídos terem sido publicados em revistas internacionais pode ser justificado com base na questão de que a COVID-19 não teve seu início no Brasil e se tornou uma pandemia global $^{(40)}$. Podem-se propor ainda, como possíveis razões para o ocorrido, a amplitude de alcance e impacto que um trabalho apresenta quando divulgado nesse tipo de jornal/revista.

Particularmente, para o maior quantitativo de produções aqui incluídas publicadas na revista European Archives of
Oto-Rhino-Laryngology, esse achado pode ser explicado se admitido que ela representa o Jornal Oficial da Confederação Europeia de Otorrinolaringologia e Cirurgia de Cabeça e Pescoço, além da sua agilidade em disseminar informações relacionadas às ciências básicas e diagnóstico e tratamento de doenças de cabeça e pescoço em nível internacional ${ }^{(38)}$.

É possível ainda que esse fenômeno tenha ocorrido se considerado que todos os estudos incluídos nesta revisão foram desenvolvidos em países europeus e pela Europa ter-se tornado o epicentro do SARSCoV-2 $2^{(41)}$. Nesse sentido, vale mencionar que a disseminação do vírus na Europa foi marcada por uma apresentação atípica da doença, principalmente em pacientes com disfunção olfativa e gustativa ${ }^{(42)}$,.

No que se refere ao país das publicações, o destaque para a Itália pode ser fundamentado no fato de ser ela o país europeu mais afetado no início da pandemia $^{(14)}$. Para o domínio do idioma inglês entre os artigos dessa pesquisa, esse dado pode ser compreendido se concebido que essas revistas publicam seus trabalhos nessa língua e por essa ser uma das estratégias utilizadas para a internacionalização científica $^{(43)}$.

Em relação aos objetivos, apesar dos estudos apresentarem diferentes focos, como $\operatorname{determinar}^{(44)} \quad\left(\operatorname{artigo} \mathrm{n}^{\mathrm{o}}\right.$ 1), estimar a prevalência $^{(42)}$ (artigo $\mathrm{n}^{\mathrm{o}}$ 2), identificar ${ }^{(45)}$ 
(artigo $\left.\mathrm{n}^{0} 4\right)$, caracterizar $^{(38)}\left(\operatorname{artigo} \mathrm{n}^{\circ} 5\right)$ e estabelecer o tempo de recuperação e gravidade dessas manifestações e associá-las aos sintomas clássicos da COVID-19(35) (artigo $n^{0} 6$ ), percebe-se a real necessidade de compreender a relação entre essas manifestações e a infecção por SARS-CoV2. Ainda, foi perceptível que a disfunção olfativa e gustativa se destacou entre os objetivos das pesquisas $^{(35,36,38,42)}$.

No que diz respeito ao tipo de estudo, houve predomínio de pesquisas descritivas. Esse resultado pode ser justificado pelo caráter atual e emergente da doença, uma vez que pesquisas descritivas têm por objetivo determinar a distribuição de condições relacionadas à saúde, de forma a descrever um determinado fenômeno com base em suas características ${ }^{(46)}$. Apesar da sua importância, esse tipo de estudo não objetiva avaliar uma possível relação entre a causa da doença e seu efeito ${ }^{(47)}$.

Com respeito ao nível de evidência, todos os estudos foram classificados com nível de evidência baixo, sinalizando para a necessidade de novas pesquisas com maior rigor metodológico sobre as manifestações neurológicas relacionadas à infecção por SARS-CoV-2.

Ao analisar os principais resultados dos estudos, observou-se que os pacientes infectados por SARS-CoV-2 apresentaram, como manifestações neurológicas mais recorrentes, a disfunção olfativa e gustativa (artigos $\mathrm{n}^{0} 2$ - 6). Nesse contexto, vale ressaltar que a disfunção olfativa é uma alteração patológica caracterizada pela incapacidade parcial ou total de detectar ou distinguir substâncias químicas voláteis ${ }^{(48)}$. Ela é multifatorial, podendo estar relacionada a doenças nasais e sinusais obstrutivas, infecções no trato respiratório superior, traumatismo cranioencefálico, envelhecimento, exposição a tóxicos e algumas medicações, neoplasias nasais ou intracranianas, patologias psiquiátricas, iatrogenia e causas idiopáticas ou congênitas $^{(49)}$.

Sobre a disfunção gustativa, essa é caracterizada pela perda ou diminuição da gustação, por meio da interação entre fatores multicausais e receptores sensitivos da cavidade oral $^{(49)}$. Além das papilas gustativas, a disfunção de paladar está diretamente relacionada a receptores químicos, mecanorreceptores, termorreceptores e nociceptores $^{(50)}$.

Com respeito aos mecanismos pelos quais o SARS-CoV-2 age interferindo no sistema neurológico dos pacientes infectados, sugerem-se duas principais vias de transmissão, a saber: a disseminação hematogênica e a disseminação retrógrada neuronal. A primeira é secundária a uma viremia, a qual desencadeia Síndrome da Resposta Inflamatória Sistêmica (SRIS). Essa 
favorece mecanismos de penetração intracelular, paracelular e transcelular através da barreira hematoencefálica. Na segunda, a invasão do SNC ocorre via transporte dos neurônios periféricos invadidos pelo vírus ${ }^{(4)}$.

Especificamente, para a disfunção olfativa e gustativa, a literatura não é clara quanto aos mecanismos patofisiológicos. Segundo Meini et al. ${ }^{(36)}$ (artigo $\mathrm{n}^{\mathrm{o}}$ 3), SARSCoV-2 infecta as células hospedeiras, incluindo as células não neuronais do epitélio olfatório e mucosa oral (inclusive a língua), pela ligação à ECA-2, aumentando os níveis de des-Arg9-bradicinina e induzindo processo inflamatório. Para a disfunção gustativa, a bradicinina pode promovê-la por meio de um mecanismo neurogênico inflamatório ou por excesso de atividade direta no $\mathrm{SNC}^{(51)}$.

Especula-se ainda que a infecção de células de suporte e células-tronco regenerativas dos neurônios olfatórios iniba a função desses últimos, desencadeando a anosmia. Outra hipótese seria a infecção do SNC pelo coronavírus, via placa cribiforme, promovendo uma resposta inflamatória capaz de reduzir a sensibilidade ao cheiro ${ }^{(52)}$.

Para a disgeusia, especula-se que níveis elevados de citocinas pró-inflamatórias, como fator de necrose tumoral $-\alpha(\mathrm{TNF}-\alpha)$, interferon $-\gamma($ INF- $\gamma)$ e interleucina -6 (IL6), induzidos pela COVID-19, inibiriam a proliferação de células-tronco e diminuiriam a meia-vida das células maduras das papilas gustativas $^{(52)}$.

Dessa forma, a disfunção olfativa e gustativa pode ser considerada um marcador de infecção por SARS-CoV-2, principalmente em situações em que a capacidade de teste é $\operatorname{limitada}^{(35)}$.

Além dessas manifestações, as pesquisas incluídas revelaram que os pacientes infectados por SARS-CoV-2 podiam apresentar dor de cabeça recorrente (artigos $n^{\circ} 1$ e 4), comprometimento de sono $\left(\operatorname{artigo} \mathrm{n}^{\circ} 4\right)$, mialgia e tontura $\left(\operatorname{artigo} \mathrm{n}^{\circ} 1\right)$. Para a cefaleia, este sintoma pode estar relacionado ao aumento de permeabilidade da barreira hematoencefálica pela produção de citocinas, desencadeando desordem neuronal de caráter inflamatório ${ }^{(44,53)}$. Poderia decorrer também da invasão direta das terminações nervosas do nervo trigêmeo na cavidade nasal pelo vírus, resultando em desregulação da ECA-2 e angiotensina $\mathrm{II}^{(54)}$.

No tocante à mialgia, essa pode ser ocasionada pela intensa resposta inflamatória induzida pelo SARS-CoV-2, a qual aumenta os níveis de citocinas, incluindo IL-6, cuja regulação positiva causa dor muscular e $\operatorname{articular}^{(55)}$. Outra hipótese associada à mialgia se baseia na elevação da lactato desidrogenase, em decorrência do dano produzido pelo vírus no tecido muscular, e do lactato e diminuição do $\mathrm{pH}$ citoplasmático e dos níveis de oxigênio ${ }^{(56)}$. Para a tontura, a 
literatura aponta, como possíveis mecanismos, a invasão direta do sistema nervoso, hipóxia, hipercoagulopatia e insultos imunomediados ${ }^{(57)}$.

Dentre outras manifestações neurológicas apresentadas pelos artigos, a disautonomia, conceituada como a falha ou hiperatividade do Sistema Nervoso Autônomo $(\mathrm{SNA})^{(58)}$, quando associada à instabilidade hemodinâmica em pacientes gravemente acometidos por COVID-19, pode resultar de uma insuficiência barorreflexa aferente secundária à infecção por SARS-CoV-2 e invasão do núcleo do trato solitário ${ }^{(58)}$.

Sobre as doenças cerebrovasculares, o acidente vascular cerebral (AVC) isquêmico, embora os mecanismos envolvidos ainda não estejam claros, tem sido associado a um estado de hipercoagulabilidade ${ }^{(59)}$. Quanto à hemorragia intracraniana, essa tem sido relacionada à elevação da pressão sanguínea como consequência da ligação do SARSCoV-2 à ECA-2 ${ }^{(53)}$.

Para a convulsão, ela pode decorrer da hipóxia, distúrbios metabólicos, falência de órgãos e dano cerebral ${ }^{(60)}$. Sobre a encefalite, caracterizada por lesões inflamatórias no cérebro $^{(61)}$, embora seu mecanismo não esteja esclarecido, sugere-se que ela é oriunda de uma resposta inflamatória ao SARS-CoV-2, capaz de elevar os níveis de interleucinas e ECA no líquido cefalorraquidiano ${ }^{(62)}$.
Com relação à neurite óptica, essa pode ocorrer se considerado que o SARSCoV-2 pode ser transportado pelo nervo óptico ${ }^{(63)}$. Quanto à Síndrome de GuillainBarré, tida como um processo autoimune que acomete os nervos periféricos e induz uma neuropatia desmielinizante ${ }^{(64)}$, a hipótese de Morsy $^{(65)}$ sugere que a proteína NCAM (molécula de adesão celular neuronal) é responsável pelo desenvolvimento da síndrome em pacientes com SARS-CoV-2, na presença de HLA-A*68 e HLA-DQA1/HLADQB1.

No tocante à miopatia, observada em pacientes com a forma grave da COVID-19, seu desenvolvimento tem sido atribuído à injúria dos nervos periféricos e músculo estriado pelo vírus, assim como pela presença de eventos vasculares, trombóticos e isquêmicos ${ }^{(66)}$.

Com respeito aos distúrbios de movimento, quando sentado (movimentos torcidos de membro inferior), caminhando (marcha atáxica) ou em repouso (movimentos espasmódicos em posição supina), pode expressar um acometimento do SNC e SNP. Quanto ao comprometimento do sono, ele pode estar associado à hospitalização, fenômeno capaz de desencadear sintomas de insônia $^{(67-68)}$.

No tocante a limitações do estudo, pode-se mencionar o reduzido número de publicações incluídas nessa revisão, apesar de 
uma busca abrangente em quatro bases de dados de relevância internacional. Esse fenômeno sugere a necessidade da condução de mais pesquisas sobre o tema abordado.

\section{CONCLUSÕES}

Conclui-se que as manifestações neurológicas estão presentes em pacientes com SARS-CoV-2/COVID-19, incluindo aqueles não hospitalizados, destacando as disfunções olfativas e gustativas. Essas podem estar associadas a um quadro de COVID-19 precoce e severo, sendo, portanto, importante o seu reconhecimento na infecção.

Além dessas disfunções, outros sinais e sintomas neurológicos, como mialgia, cefaleia, tontura e doenças cerebrovasculares, são observados em pacientes com COVID-19. Independentemente dessa forma de apresentação da doença, ela deve ser considerada, já que é capaz de comprometer o bem-estar e a vida do ser humano.

Apesar da ocorrência de manifestações neurológicas induzidas pelo SARS-CoV-2, ainda se faz necessária a investigação dos mecanismos patogênicos e progressão da doença no contexto do SNC e SNP.

\section{REFERÊNCIAS}

1. Gabriela A, Gicovate P, Leal A, Marins P, Gicovate C Neto, Shimoda E. Indicadores bibliométricos dos artigos sobre "COVID-19" na base Scopus.
Revista Científica da Faculdade de Medicina de Campos [Internet]. 2020 [Acesso em 6 nov 2020];15(2):15-21. Disponível em: http://www.fmc.br/ojs/index.php/RCF MC/article/view/383

2. Zhu N, Zhang D, Wang W, Li X, Yang $\mathrm{B}$, Song $\mathrm{J}$, et al. A novel coronavirus from patients with pneumonia in China, 2019. N Engl J Med. 2020;382(8):727-33.

3. Moitinho MS, Belasco AGS, Barbosa DA, Fonseca CD. Acute kidney injury by SARS-CoV-2 virus in patients with COVID-19: an integrative review. Revista Brasileira de Enfermagem [Internet]. 2020 [Acesso em 6 nov 2020];73(2):e20200354. Disponível em:

http://www.scielo.br/scielo.php?script= sci_arttext\&pid=S0034-

$71672020001400300 \& \operatorname{lng}=\mathrm{en} \& \mathrm{nrm}=\mathrm{is}$ o\&tlng=en

4. Alshebri MS, Alshouimi RA, Alhumidi HA, Alshaya AI. Neurological complications of SARS-CoV, MERSCoV, and COVID-19. SN Compr Clin Med [Internet]. 2020 [Acesso em 6 nov 2020];1-11. Disponível em: https://doi.org/10.1007/s42399-02000589-2

5. Nascimento Junior JAC, Santos AM, Quintans-Júnior LJ, Walker CIB, Borges LP, Serafini MR. SARS, MERS and SARS-CoV-2 (COVID-19) treatment: a patent review [Internet]. Expert Opinion on Ther Pat [Internet]. 2020 [Acesso em 6 nov 2020]; 30(8):567-79. Disponível em: https://www.tandfonline.com/doi/full/1 0.1080/13543776.2020.1772231

6. Zhong NS, Zheng BJ, Li YM, Poon LLM, Xie ZH, Chan KH, et al. Epidemiology and cause of severe acute respiratory syndrome (SARS) in Guangdong, People's Republic of 
China, in February, 2003. Lancet [Internet]. 2003 [Acesso em 6 nov 2020];362(9393):1353-8. Disponível em:

https://www.thelancet.com/journals/lan cet/article/PIIS0140-6736(03)14630-

2/fulltext

7. Guarner J. Three emerging coronaviruses in two decades: the story of SARS, MERS, and now COVID-19. American Journal of Clinical Pathology [Internet]. 2020 [Acesso em 6 nov 2020];153:420-21. Disponível em:

https://www.ncbi.nlm.nih.gov/pmc/arti cles/PMC7109697/

8. Peeri NC, Shrestha N, Rahman MS, Zaki R, Tan Z, Bibi S, et al. The SARS, MERS and novel coronavirus (COVID-19) epidemics, the newest and biggest global health threats: what lessons have we learned? Int J Epidemiol [Internet]. 2020 [Acesso em 6 nov 2020];49(3):717-26. Disponível em:

https://academic.oup.com/ije/article/49/ 3/717/5748175

9. Zumla A, Hui DS, Perlman S. Middle East respiratory syndrome. Lancet [Internet]. 2015 [Acesso em 6 nov 2020]; 386(9997):995-1007. Disponível em: https://www.thelancet.com/journals/lan cet/article/PIIS0140-6736(15)604548/fulltext

10. Badawi A, Ryoo SG. Prevalence of comorbidities in the Middle East respiratory syndrome coronavirus (MERS-CoV): a systematic review and meta-analysis. Int $J$ Infect Dis [Internet]. 2016 [Acesso em 6 nov 2020]; 49:129-33. Disponível em: https://www.ncbi.nlm.nih.gov/pmc/arti cles/PMC7110556/

11. Achar A, Ghosh C. COVID-19associated neurological disorders: the potential route of CNS invasion and blood-brain barrier relevance. Cells [Internet]. 2020 [Acesso em 6 nov 2020];9(11):2360. Disponível em: https://www.mdpi.com/2073-

$4409 / 9 / 11 / 2360$

12. Velavan TP, Meyer CG. The COVID-19 epidemic. Trop Med Int Heal [Internet]. 2020 [Acesso em 6 nov 2020];25(3):278-80. Disponível: https://onlinelibrary.wiley.com/doi/abs/ 10.1111/tmi.13383

13. Rasmussen AL. On the origins of SARS-CoV-2. Nature Medicine [Internet]. 2021 [Acesso em 26 abr 2021]:27:8-9. Disponível em: https://www.nature.com/articles/s4159 1-020-01205-5

14. World Health Organization. WHO coronavirus disease (COVID-2019) dashboard [Internet]. WHO 2021 [Acesso em 29 abr 2021]. Disponível em: https://covid19.who.int/

15. Zhang RH, Ai X, Liu Y, Li CH, Zhang HL. Genomic characterization and phylogenetic evolution of the SARSCoV-2. Acta Virol. 2020 [Acesso em 29 set 2021];64(4):496-500. Disponível em:

https://pubmed.ncbi.nlm.nih.gov/32985 209/

16. Zhu N, Zhang D, Wang W, Li X, Yang $\mathrm{B}$, Song $\mathrm{J}$, et al. A novel coronavirus from patients with pneumonia in China, 2019. N Engl J Med [Internet]. 2020 [Acesso em 6 nov 2020];382(8):727-33. Disponível em: https://www.nejm.org/doi/full/10.1056/ nejmoa2001017

17. Tammaro A, Adebanjo GAR, Parisella FR, Pezzuto A, Rello J. Cutaneous manifestations in COVID-19: the experiences of Barcelona and Rome. Journal of The European Academy of Dermatology and Venereology [Internet]. 2020 [Acesso em 6 nov 
2020];34(7):e306-7. Disponível em: https://onlinelibrary.wiley.com/doi/abs/ $10.1111 / j d v .16530$

18. Shende P, Gaikwad P, Gandhewar M, Ukey P, Bhide A, Patel V, et al. Persistence of SARS-CoV-2 in the first trimester placenta leading to transplacental transmission and fetal demise from an asymptomatic mother. Hum Reprod [Internet]. 2021 [Acesso em 29 abr 2021];36(4): 899-906. Disponível em: https://doi.org/10.1093/humrep/deaa36 7

19. Baig AM, Khaleeq A, Ali U, Syeda H. Evidence of the COVID-19 virus targeting the CNS: tissue distribution, host-virus interaction, and proposed neurotropic mechanisms. ACS Chem Neurosci [Internet]. 2020 [Acesso em 6 nov 2020];11(7):995-8. Disponível em:

https://pubs.acs.org/doi/10.1021/acsche mneuro.0c00122

20. Mao L, Jin H, Wang M, Hu Y, Chen S, $\mathrm{He} \mathrm{Q}$, et al. Neurologic manifestations of hospitalized patients with coronavirus disease 2019 in Wuhan, China. JAMA Neurol [Internet]. 2020 [Acesso em 6 nov 2020];77(6):683-90. Disponível em: https://jamanetwork.com/journals/jama neurology/fullarticle/2764549

21. Weill $\mathrm{P}$, Plissonneau $\mathrm{C}$, Legrand $\mathrm{P}$, Rioux V, Thibault R. May omega-3 fatty acid dietary supplementation help reduce severe complications in Covid19 patients? Biochimie [Internet]. 2020 [Acesso em 6 nov 2020];179:275-80. Disponíve em: https://www.ncbi.nlm.nih.gov/pmc/arti cles/PMC7481803/

22. Ng SC, Tilg H. COVID-19 and the gastrointestinal tract: more than meets the eye Gut [Internet]. 2020 [Acesso em 6 nov 2020];69(6):973-74.
Disponível em: http://gut.bmj.com/

23. Villegas-Chiroque M. Pandemia de COVID-19: pelea o huye. Revista Experiencia em Medicina Del Hospital Regional Lamabyeque [Internet]. 2020 [Acesso em 6 nov 2020];6(1):3-4. Disponível em: http://rem.hrlamb.gob.pe/index.php/RE M/article/view/424

24. Yavarpour-Bali H, Ghasemi-Kasman M. Update on neurological manifestations of COVID-19. Life Sci [Internet]. 2020 [Acesso em 6 nov 2020]; 257:1-8. Disponível em: https://www.ncbi.nlm.nih.gov/pmc/arti cles/PMC7346808/

25. Cani I, Barone V, D’Angelo R, Pisani L, Allegri V, Spinardi L, et al. Frontal encephalopathy related to hyperinflammation in COVID-19. Journal of Neurology [Internet]. 2020 [Acesso em 6 nov 2020];268(1):16-19. Disponível em: https://doi.org/10.1007/s00415-02010057-5

26. Stafstrom CE, Jantzie LL. COVID-19: neurological considerations in neonates and children. Children [Internet]. 2020 [Acesso em 6 nov 2020];7(9):133. Disponível em: https://pubmed.ncbi.nlm.nih.gov/32927 628/

27. Pereira J, Lima KMSG, Santos SMM, Silva AC, Silva DA, Farias PA, et al. Desafios da enfermagem no enfrentamento ao COVID-19. Brazilian Journal of Development [Internet]. 2021 [Acesso em 29 abr 2021];7(2):14839-55. Disponível em: https://www.brazilianjournals.com/inde x.php/BRJD/article/view/24568

28. Pezzini A, Padovani A. Lifting the mask on neurological manifestations of COVID-19. Nat Rev Neurol [Internet]. 2020 [Acesso em 6 nov 2020];16:63644. 
https://www.nature.com/articles/s4158 2-020-0398-3

29. Mendes KDS, Silveira RCCP, Galvão CM. Revisão integrativa: método de pesquisa para a incorporação de evidências na saúde e na enfermagem. Texto \& Contexto - Enfermagem [Internet]. 2008 [Acesso em 7 nov 2020];17(4):758-64. Disponível em: http://www.scielo.br/scielo.php?script= sci_arttext\&pid=S0104-

$07072008000400018 \& \ln \mathrm{g}=\mathrm{e} n \& \mathrm{nrm}=\mathrm{is}$ o\&tlng=pt

30. Sousa LMM, Marques-Vieira CMA, Severino SSP, Antunes AV. A metodologia de revisão integrativa da literatura em enfermagem. Revista Investigação em Enfermagem. [Internet]. 2017 [Acesso em 6 nov 2020];21:17-26. Disponível em: https://www.sinaisvitais.pt/index.php/r evista-investigacao-enfermagem/rieserie-2/774-rie-21-novembro-2017

31. Santos CMC, Pimenta CAM, Nobre MRC. The PICO strategy for the research question construction and evidence search. Rev Lat Am Enfermagem [Internet]. 2007 [Acesso em 6 nov 2020];15(3):508-11. Disponível em: http://www.scielo.br/scielo.php?script= sci_arttext\&pid=S0104-

$11692007000300023 \& \operatorname{lng}=$ en $\&$ tlng $=$ en

32. Moher D, Liberati A, Tetzlaff J, Altman DG, Altman D, Antes G, et al. Preferred reporting items for systematic reviews and meta-analyses: The PRISMA statement. PLoS Medicine [Internet]. 2009 [Acesso em 6 nov 2020];6(7): e1000097. Disponível em: https://dx.plos.org/10.1371/journal.pme d. 1000097

33. Ursi ES, Gavão CM. Prevenção de lesões de pele no perioperatório: revisão integrativa da literatura. Rev
Lat Am Enfermagem [Internet]. 2006 [Acesso em 6 nov 2020];14(1):124-31. Disponível em: https://www.scielo.br/scielo.php?script =sci_abstract\&pid=S0104$11692006000100017 \&$ tlng $=\mathrm{pt}$

34. Stillwell SB, Fineout-Overholt E, Melnyk BM, Williamson KM. Evidence-based practice, step by step: searching for the evidence. Am J Nurs [Internet]. 2010 [Acesso em 7 nov 2020];110(5):41-47. Disponível em: http://journals.lww.com/00000446201005000-00024

35. Speth MM, Singer-Cornelius T, Oberle M, Gengler I, Brockmeier SJ, Sedaghat AR. Olfactory dysfunction and sinonasal symptomatology in COVID19: prevalence, severity, timing, and associated characteristics. Otolaryngol Head Neck Surg [Internet]. 2020 [Acesso em 6 nov 2020];163(1):114$20 . \quad$ Disponível em: http://journals.sagepub.com/doi/10.117 7/0194599820929185

36. Meini S, Suardi LR, Busoni M, Roberts AT, Fortini A. Olfactory and gustatory dysfunctions in 100 patients hospitalized for COVID-19: sex differences and recovery time in reallife. Eur Arch Otorhinolaryngol [Internet]. 2020 [Acesso em 6 nov 2020];277(12):3519-23. Disponível em:

http://link.springer.com/10.1007/s0040 5-020-06102-8

37. Karuppan MKM, Devadoss D, Nair M, Chand HS, Lakshmana. SARS-CoV-2 infection in the central and peripheral nervous system-associated morbidities and their potencial mechanism. Mol Neurobiol [Internet]. 2021 [Acesso em 5 mai 2021]. Disponível em: https://link.springer.com/article/10.100 7/s12035-020-02245-1

38. Lechien JR, Chiesa-Estomba CM, Siati 
DR, Horoid M, Le BSD, Alexandra R, et al. Olfactory and gustatory dysfunctions as a clinical presentation of mild-to-moderate forms of the coronavirus disease (COVID-19): a multicenter European study. Eur Arch Otorhinolaryngol. [Internet]. 2020 [Acesso em 6 nov 2020];277:2251-61. Disponível em: https://link.springer.com/article/10.100 7/s00405-020-05965-1

39. Grewal A, Kataria H, Dhawan I. Literature search for research planning and identification of research problem. Indian J Anesth [Internet]. 2016 [Acesso em 6 nov 2020];60(9):635-39. Disponível em: /pmc/articles/PMC5037943/?report=ab stract

40. Dryhurst S, Schneider CR, Kerr J, Freeman ALJ, Recchia G, van der Bles AM, et al. Risk perceptions of COVID19 around the world. J Risk Res [Internet]. 2020 [Acesso em 6 nov 2020];23(7-8):994-1006. Disponível em:

https://www.tandfonline.com/doi/full/1 0.1080/13669877.2020.1758193

41. Ceylan Z. Estimation of COVID-19 prevalence in Italy, Spain, and France. Sci Total Environ [Internet]. 2020 [Acesso em 6 nov 2020];729:138817. Disponível em: https://linkinghub.elsevier.com/retrieve /pii/S0048969720323342

42. Boscolo-Rizzo P, Borsetto D, Spinato G, Fabbris C, Menegaldo A, Gaudioso $P$, et al. New onset of loss of smell or taste in household contacts of homeisolated SARS-CoV-2-positive subjects. Eur Arch Otorhinolaryngol [Internet]. 2020 [Acesso em 6 nov 2020];277(9):2637-40. Disponível em: http://link.springer.com/10.1007/s0040 5-020-06066-9

43. Cintra PR, Silva MDP, Furnival AC.
Uso do inglês como estratégia de internacionalização da produção científica em Ciências Sociais Aplicadas: estudo de caso na SciELO Brasil. Em Questão [Intenet]. 2020 [Acesso em 6 nov 2020]26(1):17-41. Disponível em: https://www.researchgate.net/publicati on/338038496_Uso_do_ingles_como_ estrategia_de_internacionalizacao_da_ producao_cientifica_em_Ciencias_Soci ais_Aplicadas_estudo_de_caso_na_Sci ELO_Brasil

44. Romero-Sánchez CM, Díaz-Maroto I, Fernández-Díaz E, Sánchez-Larsen Á, Layos-Romero A, García-García J, et al. Neurologic manifestations in hospitalized patients with COVID-19. Neurology [Internet]. 2020 [Acesso em 6 nov 2020];95(8):e1060-70. Disponível em: http://www.neurology.org/lookup/doi/1 0.1212/WNL.0000000000009937

45. Liguori C, Pierantozzi M, Spanetta M, Sarmati L, Cesta N, Iannetta M, et al. Subjective neurological symptoms frequently occur in patients with SARS-CoV2 infection. Brain Behav Immun [Internet]. 2020 [Acesso em 6 nov 2020];88:11-16. Disponível em: https://linkinghub.elsevier.com/retrieve /pii/S088915912030876X

46. Lima-Costa MF, Barreto SM. Tipos de estudos epidemiológicos: conceitos básicos e aplicações na área do envelhecimento. Epidemiol Serv Saúde [Internet]. 2003 [Acesso em 6 nov 2020];12(4):189-201. Disponível em: http://scielo.iec.pa.gov.br/scielo.php?sc ript=sci_arttext\&pid=S1679-

$49742003000400003 \& \operatorname{lng}=$ pt\&nrm $=$ is o\&tlng $=\mathrm{pt}$

47. Oliveira MA, Vellarde GC, Sá RAM. Entendendo a pesquisa clínica III: estudos de coorte [Internet]. FEMINA. 2015 [Acesso em 6 nov 2020];43(3):105-10. Disponível em: 
http://files.bvs.br/upload/S/0100-

7254/2015/v43n3/a5116.pdf

48. Hsieh JJ, Chen D, Wang PI, Marker M, Redzematovic A, Chen Y-B, et al. Genomic biomarkers of a randomized trial comparing first-line everolimus and sunitinib in patients with metastatic renal cell carcinoma. Eur Urol [Internet]. 2017 [Acesso em 6 nov 2020];71:405-14. Disponível em: https://linkinghub.elsevier.com/retrieve /pii/S0302283816307011

49. Barros JA Neto, Machado AS, Kraychete DC, Jesus RP. Comprometimento da integridade intestinal na fibromialgia e síndrome dolorosa miofascial: uma revisão. Rev Ciênc Med Biol [Internet]. 2011 [Acesso em 6 nov 2020];10(3):246-53. Disponível em https://periodicos.ufba.br/index.php/cm bio/article/view/5884\#: :text=Passos\% 20de\%20Jesus-

,Resumo,em\%20resposta\%20a\%20mec anismos\%20estressores.

50. Lahne J. Neurogastronomy: how the brain creates flavors and why it matters: by Gordon M. Shepherd. Food, Culture \& Society [Internet]. 2013 [Acesso em 6 nov 2020];16(2):327-30. Disponível em: https://books.google.com.br/books?hl= pt-

$\mathrm{BR} \& 1 \mathrm{r}=\& \mathrm{id}=\mathrm{gEigoDUBvA} 4 \mathrm{C} \& \mathrm{oi}=\mathrm{fnd}$ $\& p g=P R 7 \& d q=$ Shepherd+GM.+Neuro gastronomy.+How+the+brain+creates+ flavor+and+why+it+matters.+New+Yo rk:+Columbia+University+Press\%3B+ 2012.\&ots=EDDJcaT53D\&sig=JuGuq DNagI4zoUY3jB5d8uO_hFg\&redi

51. Belowska-Bień K, Szeląg E, Szeląg J, Skrzypiec-Spring M. Bradykinin-an undervalued mediator? Postepy Nauk Medycznych. 2017; 440-46.

52. Eshraghi AA, Mirsaeidi M, Davies C, Telischi FF, Chaudhari N, Mittal R.
Potential mechanisms for COVID-19 induced anosmia and dysgeusia. Front Physiol [Internet]. 2020 [Acesso em 7 nov 2020];11:1039. Disponível em: https://www.frontiersin.org/article/10.3 389/fphys.2020.01039/full

53. Poyiadji N, Shahin G, Noujaim D, Stone M, Patel S, Griffith B. COVID19-associated acute hemorrhagic necrotizing encephalopathy: imaging features. Radiology [Internet]. 2020 [Acesso em 6 nov 2020];296(2):E11920. Disponível em: https://doi.org/10.1148/radiol.2020201 187.

54. Bolay H, Gül A, Baykan B. COVID-19 is a real headache! Headache [Internet]. 2020 [Acesso em 6 nov 2020];60(7):1415-21. Disponível em: https://onlinelibrary.wiley.com/doi/abs/ 10.1111/head.13856

55. Drożdżal S, Rosik J, Lechowicz K, Machaj F, Szostak B, Majewski P, et al. COVID-19: pain management in patients with SARS-CoV-2 infection molecular mechanisms, challenges, and perspectives. Brain Sci [Internet]. 2020 [Acesso em 6 nov 2020];10(7):465. Disponível em: https://www.mdpi.com/2076$3425 / 10 / 7 / 465$

56. Kucuk A, Cumhur Cure M, Cure E. Can COVID-19 cause myalgia with a completely different mechanism? a hypothesis. Clin Rheumatol [Internet]. 2020 [Acesso em 6 nov 2020];39(7):2103-04. Disponível em: https://www.ncbi.nlm.nih.gov/pmc/arti cles/PMC7249985/

57. Saniasiaya J, Kulasegarah J. Dizziness and COVID-19. Ear, Nose and Throat J [Internet]. 2020 [Acesso em 6 nov 2020];100(1):29-30. Disponível em: http://www.ncbi.nlm.nih.gov/pubmed/3 2931322

58. Eshak N, Abdelnabi M, Ball S, 
Elgwairi E, Creed K, Test V, et al. Dysautonomia: an overlooked neurological manifestation in a critically ill COVID-19 patient. Am J Med Sci [Internet]. 2020 [Acesso em 6 nov 2020];360(4):427-29. Disponível em:

https://www.ncbi.nlm.nih.gov/pmc/arti cles/PMC7366085/

59. Wu T, Zuo Z, Kang S, Jiang L, Luo X, Xia Z, et al. Multi-organ dysfunction in patients with COVID-19: a systematic review and meta-analysis. Aging and Dis [Internet]. 2020 [Acesso em 6 nov 2020];11(4):874-94. Disponível em: /pmc/articles/PMC7390520/?report=ab stract

60. Asadi-Pooya AA. Seizures associated with coronavirus infections. Seizure [Internet]. 2020 [Acesso em 6 nov 2020];79:49-52. Disponível em: https://www.seizurejournal.com/article/S10591311(20)30128-X/fulltext

61. Khatoon F, Prasad K, Kumar V. Neurological manifestations of COVID-19: available evidences and a new paradigma. $J$ Neurovirol [Internet]. 2020 [Acesso em 6 nov 2020];26:619-30. Disponível em: https://pubmed.ncbi.nlm.nih.gov/32839 951/

62. Bodro M, Compta Y, Llansó L, Esteller D, Doncel-Moriano A, Mesa A, et al. Increased CSF levels of IL-1 $\beta$, IL-6, and ACE in SARS-CoV-2-associated encephalitis. Neurol Neuroimmunol Neuroinflamm [Internet]. 2020 [Acesso em 6 nov 2020];7(5):e821. Disponível em:

https://www.ncbi.nlm.nih.gov/pmc/arti cles/PMC7357418/

63. Figueiredo CS, Raony Í, Giestal-deAraujo E. SARS-CoV-2 targeting the retina: host-virus interaction and possible mechanisms of viral tropism.
Ocul Immunol Inflamm [Internet]. 2020 [Acesso em 6 nov 2020];28(8):1301-04. Disponível em: https://www.tandfonline.com/doi/full/1 0.1080/09273948.2020.1799037

64. Farzi MA, Ayromlou H, Jahanbakhsh N, Bavil PH, Janzadeh A, Shayan FK. Guillain-Barré syndrome in a patient infected with SARS-CoV-2, a case report. J Neuroimmunol [Internet]. 2020 [Acesso em 6 nov 2020];346:577294. Disponível em: https://pubmed.ncbi.nlm.nih.gov/32590 $125 /$

65. Morsy S. NCAM protein and SARSCOV-2 surface proteins: in-silico hypothetical evidence for the immunopathogenesis of Guillain-Barré syndrome. Med Hypotheses [Internet]. 2020 [Acesso em 6 nov 2020];145:110342. Disponível em: https://linkinghub.elsevier.com/retrieve /pii/S0306987720328978

66. Guadarrama-Ortiz P, Choreño-Parra JA, Sánchez-Martínez CM, PachecoSánchez FJ, Rodríguez-Nava AI, García-Quintero G. Neurological aspects of SARS-CoV-2 infection: mechanisms and manifestations. Front Neurol [Internet]. 2020 [Acesso em 6 nov 2020];11:1039. Disponível em: /pmc/articles/PMC7499054/?report=ab stract

67. Piscitelli D, Perin C, Tremolizzo L, Peroni F, Cerri CG, Cornaggia CM. Functional movement disorders in a patient with COVID-19. Neurol Sci [Internet]. 2020 [Acesso em 6 nov 2020];41(9):2343-44. Disponível em: https://doi.org/10.1007/s10072-

68. Young JS, Bougeois JA, Hilty DM, Hardin KA. Sleep in hospitalized medical patients, part 1: factors affecting sleep. J Hosp Med [Internet]. 2008 [Acesso em 6 nov 2020];3(6):473-82. Disponível em: 
http://www.journalofhospitalmedicine. com/jhospmed/article/128191/sleephospitalized-medical-patients-part-1

\section{Autor correspondente}

Ana Caroline Rocha de Melo Leite, Universidade da Integração Internacional da Lusofonia Afro-Brasiliera - Campus das Auroras - Rua José Franco de Oliveira, s/n, CEP - 62.790-970 - Redenção - Ceará, fone

(85) 99168-0679 e e-mail

acarolmelo@unilab.edu.br

Submissão: 2021-05-08

Aprovado: 2021-06-10 